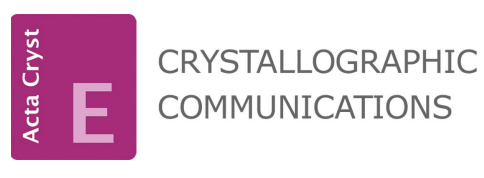

ISSN 2056-9890

Received 24 October 2017

Accepted 30 November 2017

Edited by E. V. Boldyreva, Russian Academy of Sciences, Russia

Keywords: crystal structure; flexible ligand; terephthalate; coordination polymer.

CCDC reference: 1588505

Supporting information: this article has supporting information at journals.iucr.org/e

\section{Crystal structure of a $\mathrm{Zn}$ complex with tereph- thalate and 1,6-bis(1,2,4-triazol-1-yl)hexane}

\author{
Taisiya S. Sukhikh, ${ }^{a, b *}$ Evgeny Yu. Semitut ${ }^{c, a}$ and Andrei S. Potapov ${ }^{c}$
}

\author{
aNikolaev Institute of Inorganic Chemistry, SB Russian Academy of Sciences, Akad. Lavrentiev prospekt 3, Novosibirsk \\ 90, 630090, Russian Federation, bepartment of Natural Sciences, National Research University, Novosibirsk State \\ University, Pirogova st. 2, Novosibirsk 90, 630090, Russian Federation, and ' Department of Biotechnology and Organic \\ Chemistry, National Research Tomsk Polytechnic, University, 30 Lenin Ave., 634050, Tomsk, Russian Federation. \\ *Correspondence e-mail: sukhikh@niic.nsc.ru
}

A new zinc coordination polymer with rigid benzene-1,4-dicarboxylate (bdc) and flexible 1,6-bis(1,2,4-triazol-1-yl)hexane (btrh), namely poly[[( $\mu_{2}$-benzene1,4-dicarboxylato) $\left[\mu_{2}-1,6\right.$-bis $(1,2,4$-triazol-1-yl)hexane]zinc] dimethylformamide monosolvate], $\left[\mathrm{Zn}\left(\mathrm{C}_{8} \mathrm{H}_{4} \mathrm{O}_{4}\right)\left(\mathrm{C}_{10} \mathrm{H}_{16} \mathrm{~N}_{6}\right)\right] \cdot \mathrm{C}_{3} \mathrm{H}_{7} \mathrm{NO}$, was synthesized. According to the single-crystal XRD analysis, the product crystallizes in the $P \overline{1}$ space group and has a layered structure. Analysis of the layered structure reveals $\{\mathrm{Zn}(\mathrm{bdc})\}$ chains which are connected by pairs of btrh ligands. The layers are packed tightly perpendicular to the [12 2 ] direction, separated by one nondisordered dimethylformamide solvent molecule per formula unit. According to thermogravimetric analysis, the product completely loses this solvent at $453 \mathrm{~K}$; the desolvated compound is stable up to $503 \mathrm{~K}$. As a result of the lack of hydrogen-donor groups, hydrogen bonds are not observed in the structure of the complex; however, an intermolecular $\mathrm{C}-\mathrm{H} \cdots \pi$ contact of $3.07 \AA$ occurs.

\section{Chemical context}

Coordination polymers with flexible bitopic ligands have attracted great interest as prospective materials for gas separation, sensing materials, electrochemical devices or catalysis (Pettinari et al., 2016). One of the favoured classes of bitopic ligands are bis(azol-1-yl) alkanes, which have been used for the preparation of various transition metals coordination polymers with different topologies (Alkorta et al., 2017; Pellei et al., 2017; Manzano et al., 2016; Liu et al., 2012). Bitopic bis(azol-1-yl)alkanes have two separated metalbinding sites that allow them to form a wide variety of polymeric structures. Thus, coordination compounds based on these ligands could be applied in the design of various functional materials with a wide range of potential applications. Recently, we have synthesized three new $\mathrm{Zn}$ coordination polymers based on bis(triazol-1-yl)propane and terephtalate anions (Semitut et al., 2017). By varying the conditions, it was possible to synthesize three different polymeric compounds, which have interesting luminescent properties. As part of our studies with the aim of preparing new coordination polymers with flexible bis(triazol-1-yl)alkane ligands, we report herein the synthesis and crystal structure of $[\mathrm{Zn}(\mathrm{bdc})(\mathrm{btrh})] \cdot \mathrm{DMF}$ (bdc = benzene-1,4-dicarboxylate, btrh = 1,6-bis(1,2,4-triazol1-yl)hexane, DMF = dimethylformamide). 


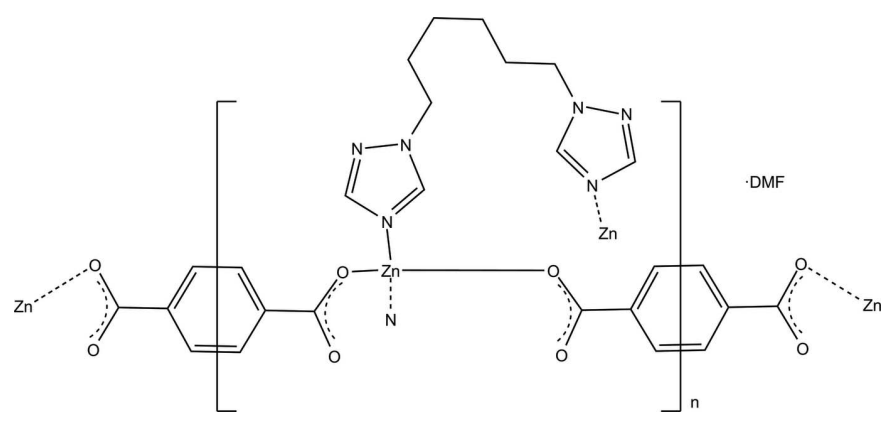

The btrh ligand (Fig. 1) was prepared by the reaction of 1,2,4-triazole with 1,6-dibromohexane in a superbasic dimethyl sulfoxide-potassium hydroxide medium using our modified procedure reported for bis(triazolyl)propane (Semitut et al., 2017). Our proposed procedure does not require the use of toxic solvents and gives higher yields compared to the literature procedure (Liu et al., 2012). The title complex was prepared by the reaction of zinc nitrate, btrh and terephthalic acid under solvothermal conditions (368 K) in DMF. The product was formed after $48 \mathrm{~h}$ as a crystalline colourless solid of plate-like shape. The single crystal used for structure determination was collected from the filtered product. The polycrystalline compound was characterized by elemental $(\mathrm{C}, \mathrm{H}, \mathrm{N})$ and powder XRD analysis (Fig. S1, Supporting information), indicating formation of this complex as a main phase.

\section{Thermal stability}

The thermal stability of the synthesized coordination polymer was studied in oxidative $\mathrm{O}_{2} / \mathrm{Ar}(21 \%)$ atmosphere. Thermogravimetric measurements were carried out on a NETZSCH thermobalance TG $209 \mathrm{~F} 1$ Iris. Open $\mathrm{Al}_{2} \mathrm{O}_{3}$ crucibles were used (loads $7-10 \mathrm{mg}$, heating rate $10 \mathrm{~K} \mathrm{~min}^{-1}$ ). The thermal analysis of $[\mathrm{Zn}(\mathrm{btrh})(\mathrm{bdc})] \cdot n \mathrm{DMF}$ revealed that the synthesized compound has three thermolysis stages in an oxidative atmosphere (Fig. 2). The first stage of thermolysis is the process of the loss of solvate molecules that runs in the range of 373-453 K and has a well-defined step on the TG curve. The mass loss of solvate molecules corresponds to a composition with $n \simeq 1$, which is in good agreement with the crystal data. The desolvated compound is stable up to $503 \mathrm{~K}$. The second and third stages run in the ranges 503-573 and 633-773 K, respectively. The second stage corresponds to partial degradation of btrh and terephtalate and third to further decomposition and the burning process of the formed carbon

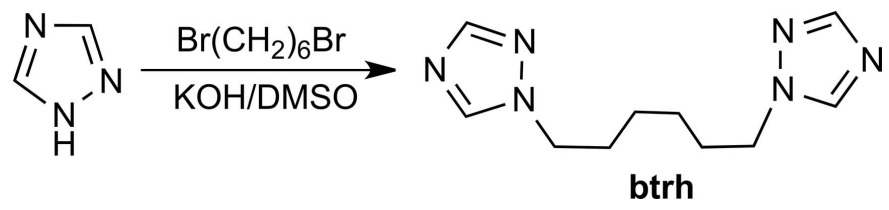

Figure 1

Synthesis of 1,6-bis(1,2,4-triazol-1-yl)hexane.

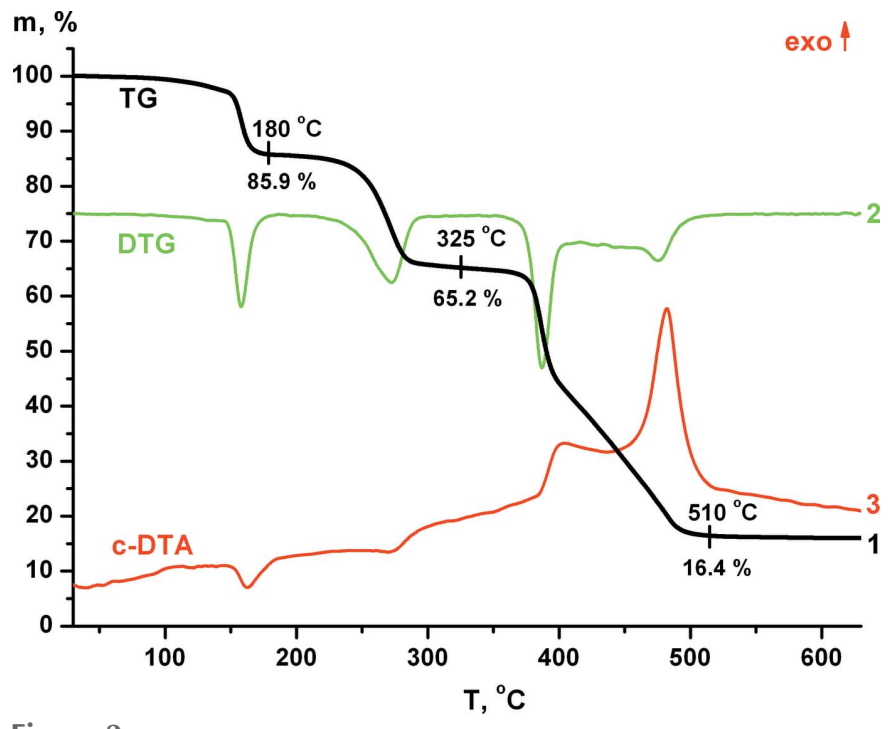

Figure 2

Curves of thermal analysis for [ $\mathrm{Zn}(\mathrm{btrh})(\mathrm{bdc})] \cdot \mathrm{DMF}$ in $\mathrm{O}_{2} / \mathrm{Ar}(21 \%)$ atmosphere; 1 TG, 2 DTG, 3 c-DTA.

products, resulting in the formation of $\mathrm{ZnO}$ according to powder XRD analysis.

\section{Structural commentary}

The structure is a $2 \mathrm{D}$ coordination polymer crystallizing in space group $P \overline{1}$. The central $\mathrm{Zn}$ atom has a distorted tetrahedral environment comprising two oxygen and two nitrogen atoms. It is coordinated by two crystallographically independent (bdc) ${ }^{2-}$ ligands (halves), forming zigzag chains along the [210] direction, which are linked by btrh ligands (Fig. 3). Contrary to our recently reported $\mathrm{Zn}$ complexes with 1,3bis(1,2,4-triazol-1-yl)propane containing a shorter alkyl bridge (Semitut et al., 2017), 1,3-bis(pyrazol-1-yl)propane (Potapov et al., 2012) and bis(imidazol-1-yl)alkanes (Barsukova, Samsonenko et al., 2016; Barsukova, Goncharova et al., 2016), the title compound is a $2 \mathrm{D}$ polymer, because the $\mathrm{Zn}$ atoms are connected by btrh ligands in pairs, not in chains, thus preventing the formation of a 3D net. Each $\mathrm{Zn}$ atom is linked with three others via (1) the first bdc ${ }^{2-}$ ligand, (2) a second $\mathrm{bdc}^{2-}$ ligand and (3) a pair of btrh ligands. The layers of the title compound are arranged perpendicular to the [122] direction in such a way that the $\left\{\mathrm{Zn}_{2}(\mathrm{btrh})_{2}\right\}$ units lie between the hollows of neighboring layers (Figs. S2, S3).

\section{Supramolecular features}

Layers of the complex are packed tightly, revealing only one DMF solvent molecule per formula unit. Analysis of the residual electron-density map clearly indicates the presence of a not or very slightly disordered DMF molecule (Fig. S4). After refining DMF, only one peak of $0.60 \mathrm{e}^{-3}$ (attributed to a $\mathrm{C}$ atom of occupancy $\mathrm{ca}$ 0.15) is observed, while the densities of other peaks coincide with those of holes $\left(c a \pm 0.3 \mathrm{e} \AA^{-3}\right)$. Thus, the DMF molecule is rather not disordered. Besides disorder, atomic displacement parameters that are larger than 
Table 1

Hydrogen-bond geometry $\left(\AA,^{\circ}\right)$.

$\mathrm{Cg}$ is the centroid of the $\mathrm{C} 24-\mathrm{C} 26 / \mathrm{C} 24^{1}-\mathrm{C} 26^{\mathrm{i}}$ ring.

\begin{tabular}{lllll}
\hline$D-\mathrm{H} \cdots A$ & $D-\mathrm{H}$ & $\mathrm{H} \cdots A$ & $D \cdots A$ & $D-\mathrm{H} \cdots A$ \\
\hline $\mathrm{C} 36^{\mathrm{ii}}-\mathrm{H} 36^{\mathrm{ii}} \cdots C g$ & 0.93 & 3.07 & 3.95 & 149 \\
\hline
\end{tabular}

Symmetry codes: (i) $2-x, 1-y,-z$; (ii) $x+1, y+1, z$.

those for other atoms can be due to partial loss of the solvent during the experiment. DMF molecules are located in the channel voids, which occupy $26.4 \%$ of the structure (Fig. S5). As a result of the lack of H-donor groups, hydrogen bonds are not observed in the structure of the complex; however, intermolecular $\mathrm{C}-\mathrm{H} \cdots \pi$ contacts of $3.07 \AA$ (Table 1) occur between the aromatic rings of bdc ligands (Fig. S6). These contacts connect neighbouring layers.

\section{Database survey}

A database survey showed that the majority of the known structures of polymers with flexible bis(azol-1-yl)alkanes are compounds based on relatively short linkers (from methane to pentane) but that the number of polymers based on longer linkers (having a $\mathrm{CH}_{2}$-chain higher than six) is relatively low. The lack of structural information on long flexible ligands can be due to the fact that it is more difficult to obtain single crystals of good quality for these compounds. Such ligands tend to form interpenetrated polymers with disorder and a variety of modifications. A search of the Cambridge Structural Database (CSD, Version 5.38, update May 2017; Groom et al.,
2016) for compounds containing btrh and any metal gave 51 hits, of which only one contains both btrh and bdc ligands (refcode ETAKAM; Zhang et al., 2011). This Cd polymer also has a 2D structure, but the $\{\mathrm{Cd}(\mathrm{bdc})\}$ chains are linear and are intersected by $\{\mathrm{Cd}(\mathrm{btrh})\}$ chains. Thus, contrary to our case, the two central metal atoms are connected by only one btrh ligand.

\section{Synthesis and crystallization}

\section{Starting materials and experimental procedures}

The starting reagents used for the synthesis of the coordination compound $-\mathrm{Zn}\left(\mathrm{NO}_{3}\right)_{2} \cdot 6 \mathrm{H}_{2} \mathrm{O}$ (chemical grade), dimethyl formamide (analytical grade) and terephthalic acid (analytical grade) - were used as received.

NMR spectra were recorded on a Bruker AV300 instrument operating at $300 \mathrm{MHz}$ for ${ }^{1} \mathrm{H}$ and $75 \mathrm{MHz}$ for ${ }^{13} \mathrm{C}$, solvent residual peaks were used as internal standard. Elemental analyses were carried out on a Eurovector EuroEA 3000 analyser. Infrared (IR) spectra of solid samples as $\mathrm{KBr}$ pellets were recorded on a FT-801 spectrometer $\left(4000-550 \mathrm{~cm}^{-1}\right)$. The powder XRD data were collected with a DRON RM4 powder diffractometer equipped with a $\mathrm{Cu} K \alpha$ source $(\lambda=$ $1.5418 \AA$ ) and graphite monochromator for the diffracted beam.

\section{Synthesis of compound [Zn(btrh)(bdc)] $\cdot n$ DMF}

$35.2 \mathrm{mg}(0.16 \mathrm{mmol})$ of btrh ligand and $4.0 \mathrm{ml}$ of $\mathrm{Zn}\left(\mathrm{NO}_{3}\right)_{2} \cdot 6 \mathrm{H}_{2} \mathrm{O}(0.04 M)$ were added to $0.4 \mathrm{ml}$ of a DMF solution of $\mathrm{H}_{2}$ bdc $(0.4 \mathrm{M})$ in a glass vial. The resulting mixture was stirred for several minutes at room temperature for total ligand dissolution and placed into an oven at $368 \mathrm{~K}$. After

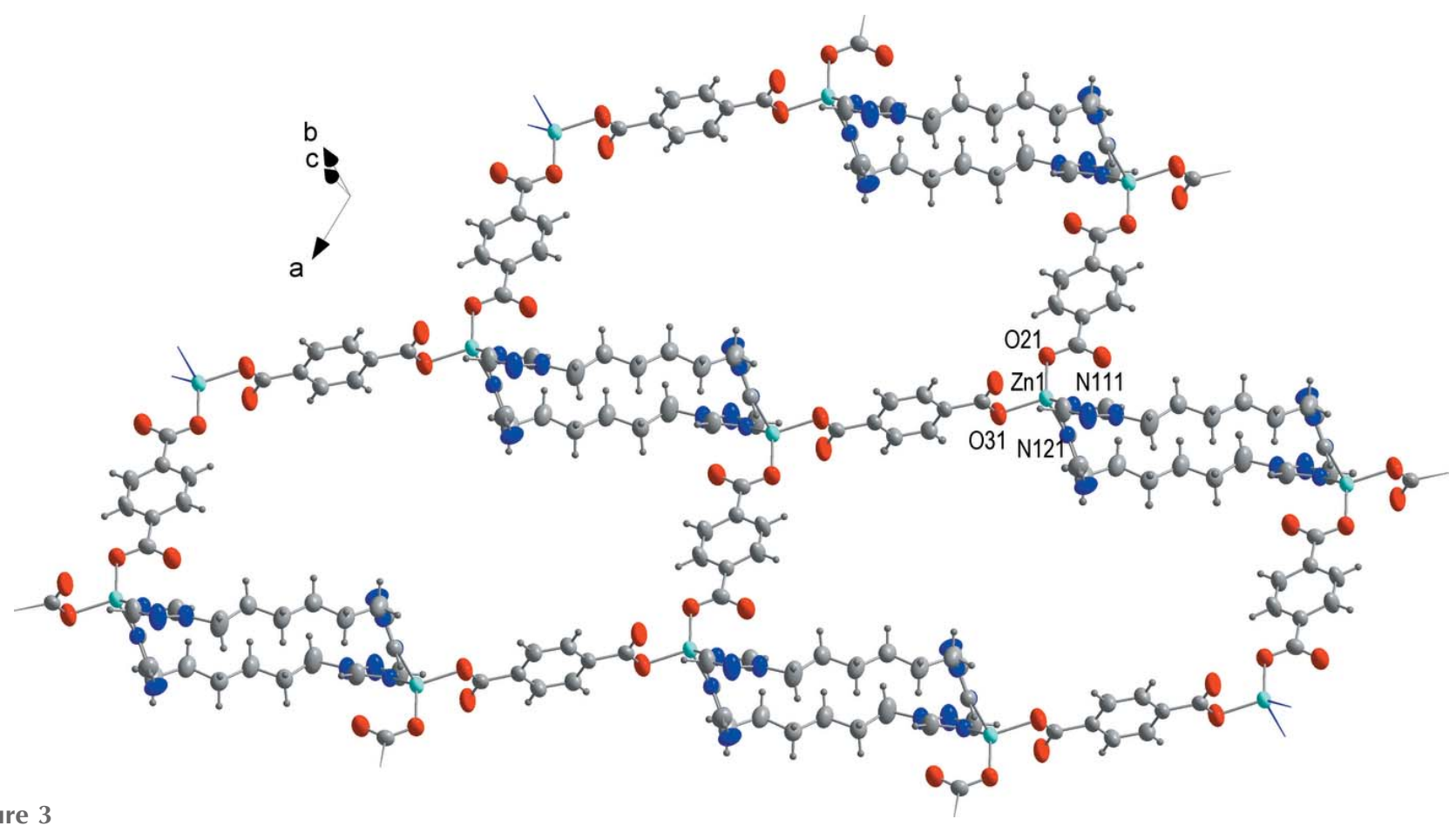

Figure 3

Displacement ellipsoid plot of a single layer of the coordination polymer showing ellispoids drawn at the $50 \%$ probability level. 
Table 2

Experimental details.

\begin{tabular}{|c|c|}
\hline \multicolumn{2}{|l|}{ Crystal data } \\
\hline Chemical formula & {$\left[\mathrm{Zn}\left(\mathrm{C}_{8} \mathrm{H}_{4} \mathrm{O}_{4}\right)\left(\mathrm{C}_{10} \mathrm{H}_{16} \mathrm{~N}_{6}\right)\right] \cdot \mathrm{C}_{3} \mathrm{H}_{7} \mathrm{NO}$} \\
\hline$M_{\mathrm{r}}$ & 522.86 \\
\hline Crystal system, space group & Triclinic, $P \overline{1}$ \\
\hline Temperature $(\mathrm{K})$ & 298 \\
\hline$a, b, c(\AA)$ & $9.7803(6), 10.4481(5), 13.3708(8)$ \\
\hline$\alpha, \beta, \gamma\left({ }^{\circ}\right)$ & $\begin{array}{l}101.438(2), 101.015(2) \\
109.073(2)\end{array}$ \\
\hline$V\left(\AA^{3}\right)$ & $1216.41(12)$ \\
\hline$Z$ & 2 \\
\hline Radiation type & Мо $K \alpha$ \\
\hline$\mu\left(\mathrm{mm}^{-1}\right)$ & 1.06 \\
\hline Crystal size $(\mathrm{mm})$ & $0.1 \times 0.05 \times 0.02$ \\
\hline \multicolumn{2}{|l|}{ Data collection } \\
\hline Diffractometer & Bruker APEXII CCD \\
\hline Absorption correction & $\begin{array}{l}\text { Multi-scan (SADABS; Bruker, } \\
\text { 2012) }\end{array}$ \\
\hline$T_{\min }, T_{\max }$ & $0.665,0.745$ \\
\hline $\begin{array}{l}\text { No. of measured, independent and } \\
\text { observed }[I>2 \sigma(I)] \text { reflections }\end{array}$ & 12157, 4293, 2999 \\
\hline$R_{\text {int }}$ & 0.049 \\
\hline$(\sin \theta / \lambda)_{\max }\left(\AA^{-1}\right)$ & 0.595 \\
\hline \multicolumn{2}{|l|}{ Refinement } \\
\hline$R\left[F^{2}>2 \sigma\left(F^{2}\right)\right], w R\left(F^{2}\right), S$ & $0.047,0.118,1.00$ \\
\hline No. of reflections & 4293 \\
\hline No. of parameters & 309 \\
\hline $\mathrm{H}$-atom treatment & H-atom parameters constrained \\
\hline$\Delta \rho_{\max }, \Delta \rho_{\min }\left(\mathrm{e} \AA^{-3}\right)$ & $0.60,-0.33$ \\
\hline
\end{tabular}

Computer programs: APEX2 and SAINT (Bruker, 2012), SHELXT (Sheldrick, 2015a), SHELXL2014 (Sheldrick, 2015b) and OLEX2 (Dolomanov et al., 2009).

heating for $48 \mathrm{~h}$, the vial was cooled to room temperature. Plate-like colourless crystals formed on the bottom of the vial; they where filtered and washed twice with $5 \mathrm{ml}$ of DMF and dried in a vacuum. The yield was $39 \mathrm{mg}$ (53\%). IR bands, $\mathrm{cm}^{-1}:$ 3115, 2948, 2861, 1680, 1611, 1530, 1499, 1437, 1391, 1345, 1287, 1217, 1136, 1098, 1017, 1001, 947, 905, 878, 828, 750, 743, 673, 642, 577. Elemental analysis: found, \%: C 48.5, H 5.9, N 18.9; calculated ([Zn(btrh)(bdc)].DMF), \%: C 48.2, H 5.2, N 18.8 .

\section{Synthesis of 1,6-bis(1,2,4-triazol-1-yl)hexane (btrh)}

A suspension of $2.76 \mathrm{~g}$ (40 mmol) of 1,2,4-triazole and $4.48 \mathrm{~g}(80 \mathrm{mmol})$ of powdered $\mathrm{KOH}$ in $15 \mathrm{ml}$ of DMSO was stirred vigorously at $353 \mathrm{~K}$ for $30 \mathrm{~min}$. The reaction flask was then immersed into a cold water bath and, after cooling to room temperature, $4.88 \mathrm{~g}(20 \mathrm{mmol})$ of 1,6-dibromohexane in $10 \mathrm{ml}$ of DMSO were added dropwise over $30 \mathrm{~min}$. After the addition was complete, the reaction mixture was stirred overnight at $353 \mathrm{~K}$. It was then quenched with $200 \mathrm{ml}$ of water and extracted with 1-butanol $(5 \times 20 \mathrm{ml})$, the extract was then washed with water $(2 \times 10 \mathrm{ml})$. Evaporation of solvents from the extract on a rotary evaporator and recrystallization from isopropyl alcohol gave $3.83 \mathrm{~g}(87 \%)$ of the product as colourless crystals. ${ }^{1} \mathrm{H}$ NMR $\left(\mathrm{CDCI}_{3}\right), \delta, \mathrm{ppm}: 1.24(t, 4 \mathrm{H}, \gamma$ $\left.\mathrm{CH}_{2}, J=7 \mathrm{~Hz}\right), 1.79\left(q, 4 \mathrm{H}, \beta-\mathrm{CH}_{2}, J=7 \mathrm{~Hz}\right), 4.06(t, 4 \mathrm{H}, \alpha-$ $\left.\mathrm{CH}_{2}, J=7 \mathrm{~Hz}\right), 7.83\left(s, 2 \mathrm{H}, \mathrm{H}^{3}-\mathrm{Tr}\right), 8.08\left(s, 2 \mathrm{H}, \mathrm{H}^{5}-\mathrm{Tr}\right) .{ }^{13} \mathrm{C}$ NMR $\left(\mathrm{CDCI}_{3}\right), \delta$, ppm: $25.6\left(\gamma-\mathrm{CH}_{2}\right), 29.3\left(\beta-\mathrm{CH}_{2}\right), 49.2(\alpha$ $\left.-\mathrm{CH}_{2}\right), 142.7\left(\mathrm{Tr}-\mathrm{C}^{3}\right), 151.6\left(\mathrm{Tr}-\mathrm{C}^{5}\right)$.

\section{Refinement}

Crystal data, data collection and structure refinement details are summarized in Table $2 . \mathrm{H}$ atoms were refined as riding atoms $\left(\mathrm{C}-\mathrm{H}=0.97 \AA\right.$ with $U_{\text {iso }}(\mathrm{H})=1.5 U_{\text {eq }}(\mathrm{C})$ for methyl $\mathrm{H}$ atoms and $\mathrm{C}-\mathrm{H}=0.93 \AA 1.2 U_{\text {eq }}(\mathrm{C})$ for all others. Methyl $\mathrm{H}$ atoms were refined as rotating groups.

\section{Funding information}

This study was supported by the Russian Science Foundation, grant No. 15-13-10023 and the X-ray structure analysis was carried out with support from the Ministry of Education and Science of the Russian Federation (a project of the Joint Laboratories of the Siberian Branch of the Russian Academy of Sciences and the National Research Universities).

\section{References}

Alkorta, I., Claramunt, R. M., Díez-Barra, E., Elguero, J., de la Hoz, A. \& López, C. (2017). Coord. Chem. Rev. 339, 153-182.

Barsukova, M., Goncharova, T., Samsonenko, D., Dybtsev, D. \& Potapov, A. (2016). Crystals, 6, 132/1-132/15.

Barsukova, M. O., Samsonenko, D. G., Goncharova, T. V., Potapov, A. S., Sapchenko, S. A., Dybtsev, D. N. \& Fedin, V. P. (2016). Russ. Chem. Bull. 65, 2914-2919.

Bruker (2014). APEX2, SAINT and SADABS. Bruker AXS Inc., Madison, Wisconsin, USA.

Dolomanov, O. V., Bourhis, L. J., Gildea, R. J., Howard, J. A. K. \& Puschmann, H. (2009). J. Appl. Cryst. 42, 339-341.

Groom, C. R., Bruno, I. J., Lightfoot, M. P. \& Ward, S. C. (2016). Acta Cryst. B72, 171-179.

Liu, Y.-Y., Li, J., Ma, J.-F., Ma, J.-C. \& Yang, J. (2012). CrystEngComm, 14, 169-177.

Manzano, B. R., Jalón, F. A., Carrión, M. C. \& Durá, G. (2016). Eur. J. Inorg. Chem. 2016, 2272-2295.

Pellei, M., Santini, C., Marinelli, M., Trasatti, A. \& Dias, H. V. R. (2017). Polyhedron, 125, 86-92.

Pettinari, C., Tăbăcaru, A. \& Galli, S. (2016). Coord. Chem. Rev. 307, $1-31$.

Potapov, A. S., Domina, G. A., Petrenko, T. V. \& Khlebnikov, A. I. (2012). Polyhedron, 33, 150-157.

Semitut, E. Y., Sukhikh, T. S., Filatov, E. Y., Anosova, G. A., Ryadun, A. A., Kovalenko, K. A. \& Potapov, A. S. (2017). Cryst. Growth Des. 17, 5559-5567.

Sheldrick, G. M. (2015a). Acta Cryst. A71, 3-8.

Sheldrick, G. M. (2015b). Acta Cryst. C71, 3-8.

Zhang, P., Li, D.-S., Zhao, J., Wu, Y.-P., Li, C., Zou, K. \& Lu, J. Y. (2011). J. Coord. Chem. 64, 13, 2329-2341. 


\section{supporting information}

Acta Cryst. (2018). E74, 6-9 [https://doi.org/10.1107/S2056989017017224]

\section{Crystal structure of a Zn complex with terephthalate and 1,6-bis(1,2,4- triazol-1-yl)hexane}

\section{Taisiya S. Sukhikh, Evgeny Yu. Semitut and Andrei S. Potapov}

Computing details

Data collection: APEX2 (Bruker, 2012); cell refinement: SAINT (Bruker, 2012); data reduction: SAINT (Bruker, 2012); program(s) used to solve structure: SHELXT (Sheldrick, 2015a); program(s) used to refine structure: SHELXL2014 (Sheldrick, 2015b); molecular graphics: OLEX2 (Dolomanov et al., 2009); software used to prepare material for publication: OLEX2 (Dolomanov et al., 2009).

Poly $\left[\left(\mu_{2}\right.\right.$-benzene-1,4-dicarboxylato $\left[\mu_{2}\right.$-1,6-bis(1,2,4-triazol-1-yl)hexane $]$ zinc $]$ dimethylformamide monosolvate]

Crystal data

$\left[\mathrm{Zn}\left(\mathrm{C}_{8} \mathrm{H}_{4} \mathrm{O}_{4}\right)\left(\mathrm{C}_{10} \mathrm{H}_{16} \mathrm{~N}_{6}\right)\right] \cdot \mathrm{C}_{3} \mathrm{H}_{7} \mathrm{NO}$

$M_{r}=522.86$

Triclinic, $P \overline{1}$

$a=9.7803(6) \AA$

$b=10.4481(5) \AA$

$c=13.3708(8) \AA$

$\alpha=101.438(2)^{\circ}$

$\beta=101.015(2)^{\circ}$

$\gamma=109.073(2)^{\circ}$

$V=1216.41(12) \AA^{3}$

Data collection

Bruker APEXII CCD

diffractometer

$\varphi$ and $\omega$ scans

Absorption correction: multi-scan

(SADABS; Bruker, 2012)

$T_{\min }=0.665, T_{\max }=0.745$

12157 measured reflections

\section{Refinement}

Refinement on $F^{2}$

Least-squares matrix: full

$R\left[F^{2}>2 \sigma\left(F^{2}\right)\right]=0.047$

$w R\left(F^{2}\right)=0.118$

$S=1.00$

4293 reflections

309 parameters

0 restraints
$Z=2$

$F(000)=544$

$D_{\mathrm{x}}=1.428 \mathrm{Mg} \mathrm{m}^{-3}$

Mo $K \alpha$ radiation, $\lambda=0.71073 \AA$

Cell parameters from 2516 reflections

$\theta=2.3-22.4^{\circ}$

$\mu=1.06 \mathrm{~mm}^{-1}$

$T=298 \mathrm{~K}$

Plate, colourless

$0.1 \times 0.05 \times 0.02 \mathrm{~mm}$

4293 independent reflections

2999 reflections with $I>2 \sigma(I)$

$R_{\text {int }}=0.049$

$\theta_{\max }=25.0^{\circ}, \theta_{\min }=2.2^{\circ}$

$h=-11 \rightarrow 11$

$k=-12 \rightarrow 9$

$l=-15 \rightarrow 15$

Hydrogen site location: inferred from neighbouring sites

$\mathrm{H}$-atom parameters constrained

$w=1 /\left[\sigma^{2}\left(F_{\mathrm{o}}^{2}\right)+(0.062 P)^{2}\right]$

where $P=\left(F_{\mathrm{o}}^{2}+2 F_{\mathrm{c}}{ }^{2}\right) / 3$

$(\Delta / \sigma)_{\max }<0.001$

$\Delta \rho_{\max }=0.60 \mathrm{e}^{-3}$

$\Delta \rho_{\min }=-0.33$ e $\AA^{-3}$ 


\section{Special details}

Geometry. All esds (except the esd in the dihedral angle between two 1.s. planes) are estimated using the full covariance matrix. The cell esds are taken into account individually in the estimation of esds in distances, angles and torsion angles; correlations between esds in cell parameters are only used when they are defined by crystal symmetry. An approximate (isotropic) treatment of cell esds is used for estimating esds involving l.s. planes.

Fractional atomic coordinates and isotropic or equivalent isotropic displacement parameters $\left(\AA^{2}\right)$

\begin{tabular}{|c|c|c|c|c|}
\hline & $x$ & $y$ & $z$ & $U_{\text {iso }} * / U_{\text {eq }}$ \\
\hline Zn1 & $0.55113(4)$ & 0.42735 & $0.19802(3)$ & $0.04413(17)$ \\
\hline $\mathrm{O} 21$ & $0.6685(3)$ & 0.4028 & $0.0983(2)$ & $0.0588(7)$ \\
\hline $\mathrm{O} 22$ & $0.8610(3)$ & $0.5441(3)$ & $0.2351(2)$ & $0.0751(9)$ \\
\hline $\mathrm{O} 31$ & 0.3399 & $0.3182(3)$ & $0.1154(3)$ & $0.0745(9)$ \\
\hline $\mathrm{O} 32$ & $0.3836(4)$ & 0.1533 & $0.1758(3)$ & $0.0911(11)$ \\
\hline N111 & $0.6118(3)$ & $0.4320(3)$ & $0.3513(2)$ & $0.0476(7)$ \\
\hline N113 & $0.6160(4)$ & $0.3855(4)$ & $0.5075(3)$ & $0.0749(11)$ \\
\hline N114 & $0.7283(4)$ & $0.5081(3)$ & $0.5193(3)$ & $0.0586(9)$ \\
\hline N121 & $1.4483(3)$ & $1.3736(3)$ & 0.7898 (2) & $0.0464(7)$ \\
\hline N123 & $1.4969(4)$ & $1.1793(4)$ & $0.7385(3)$ & $0.0701(10)$ \\
\hline N124 & $1.4569(3)$ & $1.1841(3)$ & $0.8295(3)$ & $0.0504(8)$ \\
\hline $\mathrm{C} 23$ & $0.8065(4)$ & 0.4779 (4) & $0.1401(3)$ & $0.0486(9)$ \\
\hline $\mathrm{C} 24$ & 0.9064 (4) & $0.4885(3)$ & $0.0678(3)$ & $0.0436(9)$ \\
\hline $\mathrm{C} 25$ & $1.0606(4)$ & 0.5593 & $0.1062(3)$ & $0.0654(12)$ \\
\hline $\mathrm{H} 25$ & 1.1032 & 0.6004 & 0.1788 & $0.078^{*}$ \\
\hline $\mathrm{C} 26$ & $1.1517(4)$ & $0.5703(4)$ & 0.0403 & 0.0637 (11) \\
\hline $\mathrm{H} 26$ & 1.2551 & 0.6186 & 0.0688 & $0.076^{*}$ \\
\hline $\mathrm{C} 33$ & $0.3002(4)$ & $0.1957(4)$ & $0.1235(3)$ & $0.0540(10)$ \\
\hline C34 & $0.1434(4)$ & 0.0945 (4) & $0.0602(3)$ & $0.0473(9)$ \\
\hline $\mathrm{C} 35$ & $0.0949(4)$ & $-0.0457(4)$ & $0.0597(3)$ & $0.0544(10)$ \\
\hline H35 & 0.1584 & -0.0773 & 0.1002 & $0.065^{*}$ \\
\hline C36 & $-0.0471(4)$ & -0.1389 & $-0.0003(3)$ & $0.0547(10)$ \\
\hline H36 & -0.0781 & -0.2331 & -0.0003 & $0.066^{*}$ \\
\hline C112 & $0.5502(5)$ & $0.3442(4)$ & $0.4054(4)$ & $0.0674(12)$ \\
\hline H112 & 0.4670 & 0.2605 & 0.3729 & $0.081^{*}$ \\
\hline C115 & $0.7231(5)$ & $0.5337(4)$ & $0.4267(3)$ & $0.0596(11)$ \\
\hline H115 & 0.7894 & 0.6132 & 0.4158 & $0.071^{*}$ \\
\hline $\mathrm{C} 122$ & $1.4891(5)$ & $1.2946(4)$ & $0.7177(3)$ & $0.0658(12)$ \\
\hline H122 & 1.5100 & 1.3199 & 0.6577 & $0.079^{*}$ \\
\hline $\mathrm{C} 125$ & $1.4289(4)$ & $1.2994(4)$ & $0.8595(3)$ & $0.0496(9)$ \\
\hline H125 & 1.3999 & 1.3247 & 0.9204 & $0.060^{*}$ \\
\hline C131 & $0.8254(6)$ & $0.5943(5)$ & $0.6262(3)$ & 0.0807 (14) \\
\hline H13A & 0.8279 & 0.5326 & 0.6714 & $0.097^{*}$ \\
\hline H13B & 0.7810 & 0.6584 & 0.6554 & $0.097 *$ \\
\hline C132 & $0.9823(5)$ & $0.6778(4)$ & $0.6292(3)$ & $0.0662(12)$ \\
\hline $\mathrm{H} 13 \mathrm{C}$ & 0.9812 & 0.7343 & 0.5797 & $0.079 *$ \\
\hline H13D & 1.0317 & 0.6146 & 0.6076 & $0.079 *$ \\
\hline C133 & $1.0684(5)$ & 0.7730 & $0.7397(3)$ & $0.0656(12)$ \\
\hline H13E & 1.0744 & 0.7146 & 0.7871 & $0.079 *$ \\
\hline
\end{tabular}




$\begin{array}{lllll}\text { H13F } & 1.0118 & 0.8284 & 0.7629 & 0.079^{*} \\ \text { C134 } & 1.2262(5) & 0.8728(4) & 0.7510(3) & 0.0628(11) \\ \text { H13G } & 1.2858 & 0.8186 & 0.7319 & 0.075^{*} \\ \text { H13H } & 1.2222 & 0.9302 & 0.7025 & 0.075^{*} \\ \text { C135 } & 1.3004(5) & 0.9675(4) & 0.8631(3) & 0.0636(12) \\ \text { H13I } & 1.2358 & 1.0160 & 0.8823 & 0.076^{*} \\ \text { H13J } & 1.3056 & 0.9083 & 0.9100 & 0.076^{*} \\ \text { C136 } & 1.4545(5) & 1.0756(4) & 0.8842(3) & 0.0654(12) \\ \text { H13K } & 1.4953 & 1.1204 & 0.9600 & 0.078^{*} \\ \text { H13L } & 1.5191 & 1.0290 & 0.8617 & 0.078^{*} \\ \text { O1S } & 1.2093(8) & 1.1555(6) & 0.4821(6) & 0.225(4) \\ \text { N3S } & 1.1710(7) & 0.9340(5) & 0.4102(4) & 0.1045(16) \\ \text { C2S } & 1.2272(11) & 1.0486(9) & 0.4748(8) & 0.179(4) \\ \text { H2S } & 1.3017 & 1.0532 & 0.5318 & 0.215^{*} \\ \text { C4S } & 1.1971(10) & 0.8107(8) & 0.4102(7) & 0.175(4) \\ \text { H4SA } & 1.2829 & 0.8303 & 0.4680 & 0.263^{*} \\ \text { H4SB } & 1.2159 & 0.7757 & 0.3443 & 0.263^{*} \\ \text { H4SC } & 1.1104 & 0.7411 & 0.4181 & 0.263^{*} \\ \text { C5S } & 1.0403(15) & 0.9099(11) & 0.3279(8) & 0.261(7) \\ \text { H5SA } & 0.9604 & 0.9146 & 0.3586 & 0.392^{*} \\ \text { H5SB } & 1.0094 & 0.8183 & 0.2790 & 0.392^{*} \\ \text { H5SC } & 1.0637 & 0.9807 & 0.2909 & 0.392^{*}\end{array}$

Atomic displacement parameters $\left(\AA^{2}\right)$

\begin{tabular}{lllllll}
\hline & $U^{11}$ & $U^{22}$ & $U^{33}$ & $U^{12}$ & $U^{13}$ & $U^{23}$ \\
\hline Zn1 & $0.0345(2)$ & $0.0472(3)$ & $0.0430(3)$ & $0.00801(18)$ & $0.01047(19)$ & $0.00923(18)$ \\
O21 & $0.0449(16)$ & $0.0665(17)$ & $0.0534(18)$ & $0.0082(13)$ & $0.0217(14)$ & $0.0061(13)$ \\
O22 & $0.0524(18)$ & $0.100(2)$ & $0.052(2)$ & $0.0115(16)$ & $0.0196(16)$ & $0.0013(17)$ \\
O31 & $0.0462(17)$ & $0.0557(18)$ & $0.097(3)$ & $0.0006(13)$ & $0.0069(16)$ & $0.0137(16)$ \\
O32 & $0.061(2)$ & $0.070(2)$ & $0.104(3)$ & $0.0154(16)$ & $-0.0199(19)$ & $-0.0029(18)$ \\
N111 & $0.0452(18)$ & $0.0493(17)$ & $0.0420(19)$ & $0.0111(14)$ & $0.0116(16)$ & $0.0114(15)$ \\
N113 & $0.070(3)$ & $0.078(2)$ & $0.053(3)$ & $0.000(2)$ & $0.008(2)$ & $0.0255(19)$ \\
N114 & $0.055(2)$ & $0.061(2)$ & $0.045(2)$ & $0.0058(17)$ & $0.0104(17)$ & $0.0148(16)$ \\
N121 & $0.0449(18)$ & $0.0446(17)$ & $0.0431(19)$ & $0.0117(14)$ & $0.0118(15)$ & $0.0077(15)$ \\
N123 & $0.093(3)$ & $0.069(2)$ & $0.063(3)$ & $0.041(2)$ & $0.037(2)$ & $0.0171(19)$ \\
N124 & $0.0488(19)$ & $0.0504(19)$ & $0.046(2)$ & $0.0174(15)$ & $0.0054(16)$ & $0.0093(15)$ \\
C23 & $0.045(2)$ & $0.055(2)$ & $0.051(3)$ & $0.0197(19)$ & $0.021(2)$ & $0.016(2)$ \\
C24 & $0.038(2)$ & $0.046(2)$ & $0.043(2)$ & $0.0121(16)$ & $0.0134(18)$ & $0.0102(17)$ \\
C25 & $0.042(2)$ & $0.092(3)$ & $0.040(3)$ & $0.011(2)$ & $0.008(2)$ & $-0.001(2)$ \\
C26 & $0.032(2)$ & $0.093(3)$ & $0.048(3)$ & $0.008(2)$ & $0.010(2)$ & $0.006(2)$ \\
C33 & $0.039(2)$ & $0.056(3)$ & $0.060(3)$ & $0.017(2)$ & $0.017(2)$ & $0.002(2)$ \\
C34 & $0.036(2)$ & $0.045(2)$ & $0.054(3)$ & $0.0101(16)$ & $0.0138(18)$ & $0.0059(17)$ \\
C35 & $0.041(2)$ & $0.051(2)$ & $0.065(3)$ & $0.0149(18)$ & $0.008(2)$ & $0.0133(19)$ \\
C36 & $0.048(2)$ & $0.042(2)$ & $0.068(3)$ & $0.0121(18)$ & $0.014(2)$ & $0.0144(19)$ \\
C112 & $0.061(3)$ & $0.063(3)$ & $0.058(3)$ & $-0.001(2)$ & $0.009(2)$ & $0.022(2)$ \\
C115 & $0.061(3)$ & $0.059(2)$ & $0.048(3)$ & $0.007(2)$ & $0.017(2)$ & $0.017(2)$ \\
C122 & $0.072(3)$ & $0.071(3)$ & $0.054(3)$ & $0.024(2)$ & $0.026(2)$ & $0.015(2)$
\end{tabular}




\begin{tabular}{lllllll}
\hline & & & & & \\
$\mathrm{C} 125$ & $0.044(2)$ & $0.053(2)$ & $0.047(2)$ & $0.0171(18)$ & $0.0098(18)$ & $0.0097(19)$ \\
$\mathrm{C} 131$ & $0.087(4)$ & $0.083(3)$ & $0.044(3)$ & $0.006(3)$ & $0.008(3)$ & $0.010(2)$ \\
$\mathrm{C} 132$ & $0.054(3)$ & $0.074(3)$ & $0.056(3)$ & $0.022(2)$ & $0.003(2)$ & $0.001(2)$ \\
$\mathrm{C} 133$ & $0.071(3)$ & $0.057(2)$ & $0.052(3)$ & $0.016(2)$ & $0.002(2)$ & $0.008(2)$ \\
$\mathrm{C} 134$ & $0.060(3)$ & $0.065(3)$ & $0.055(3)$ & $0.026(2)$ & $0.005(2)$ & $0.006(2)$ \\
$\mathrm{C} 135$ & $0.076(3)$ & $0.055(2)$ & $0.049(3)$ & $0.021(2)$ & $0.001(2)$ & $0.0133(19)$ \\
$\mathrm{C} 136$ & $0.077(3)$ & $0.057(2)$ & $0.054(3)$ & $0.028(2)$ & $-0.003(2)$ & $0.012(2)$ \\
O1S & $0.253(8)$ & $0.094(4)$ & $0.257(8)$ & $0.083(4)$ & $-0.056(6)$ & $-0.012(4)$ \\
N3S & $0.146(5)$ & $0.079(3)$ & $0.084(4)$ & $0.049(3)$ & $0.020(3)$ & $0.013(3)$ \\
C2S & $0.214(10)$ & $0.096(6)$ & $0.187(9)$ & $0.047(6)$ & $0.008(8)$ & $0.017(6)$ \\
C4S & $0.199(9)$ & $0.149(7)$ & $0.189(9)$ & $0.099(7)$ & $0.052(7)$ & $0.017(6)$ \\
C5S & $0.345(17)$ & $0.179(10)$ & $0.186(10)$ & $0.104(10)$ & $-0.075(12)$ & $0.026(8)$ \\
& & & & & & \\
\hline
\end{tabular}

Geometric parameters $\left(\AA,{ }^{\circ}\right)$

\begin{tabular}{|c|c|c|c|}
\hline $\mathrm{Zn} 1-\mathrm{O} 21$ & $1.950(3)$ & $\mathrm{C} 36-\mathrm{H} 36$ & 0.9300 \\
\hline $\mathrm{Zn} 1-\mathrm{O} 31$ & $1.969(3)$ & $\mathrm{C} 112-\mathrm{H} 112$ & 0.9300 \\
\hline $\mathrm{Zn} 1-\mathrm{N} 111$ & $2.008(3)$ & C115-H115 & 0.9300 \\
\hline $\mathrm{Zn} 1-\mathrm{N} 121^{\mathrm{i}}$ & $2.052(3)$ & $\mathrm{C} 122-\mathrm{H} 122$ & 0.9300 \\
\hline $\mathrm{O} 21-\mathrm{C} 23$ & $1.263(4)$ & C125-H125 & 0.9300 \\
\hline $\mathrm{O} 22-\mathrm{C} 23$ & $1.236(4)$ & $\mathrm{C} 131-\mathrm{H} 13 \mathrm{~A}$ & 0.9700 \\
\hline $\mathrm{O} 31-\mathrm{C} 33$ & $1.244(5)$ & C131-H13B & 0.9700 \\
\hline $\mathrm{O} 32-\mathrm{C} 33$ & $1.222(5)$ & $\mathrm{C} 131-\mathrm{C} 132$ & $1.485(6)$ \\
\hline $\mathrm{N} 111-\mathrm{C} 112$ & $1.340(5)$ & $\mathrm{C} 132-\mathrm{H} 13 \mathrm{C}$ & 0.9700 \\
\hline N111-C115 & $1.315(5)$ & $\mathrm{C} 132-\mathrm{H} 13 \mathrm{D}$ & 0.9700 \\
\hline N113-N114 & $1.344(4)$ & $\mathrm{C} 132-\mathrm{C} 133$ & $1.509(5)$ \\
\hline N113-C112 & $1.309(5)$ & $\mathrm{C} 133-\mathrm{H} 13 \mathrm{E}$ & 0.9700 \\
\hline N114-C115 & $1.314(5)$ & C133-H13F & 0.9700 \\
\hline N114-C131 & $1.472(5)$ & $\mathrm{C} 133-\mathrm{C} 134$ & $1.513(6)$ \\
\hline $\mathrm{N} 121-\mathrm{Zn} 1^{\mathrm{i}}$ & $2.052(3)$ & $\mathrm{C} 134-\mathrm{H} 13 \mathrm{G}$ & 0.9700 \\
\hline N121-C122 & $1.348(5)$ & $\mathrm{C} 134-\mathrm{H} 13 \mathrm{H}$ & 0.9700 \\
\hline $\mathrm{N} 121-\mathrm{C} 125$ & $1.327(5)$ & $\mathrm{C} 134-\mathrm{C} 135$ & $1.510(5)$ \\
\hline $\mathrm{N} 123-\mathrm{N} 124$ & $1.343(4)$ & C135-H13I & 0.9700 \\
\hline $\mathrm{N} 123-\mathrm{C} 122$ & $1.311(5)$ & C135-H13J & 0.9700 \\
\hline $\mathrm{N} 124-\mathrm{C} 125$ & $1.324(4)$ & $\mathrm{C} 135-\mathrm{C} 136$ & $1.492(6)$ \\
\hline $\mathrm{N} 124-\mathrm{C} 136$ & $1.462(5)$ & $\mathrm{C} 136-\mathrm{H} 13 \mathrm{~K}$ & 0.9700 \\
\hline $\mathrm{C} 23-\mathrm{C} 24$ & $1.495(5)$ & $\mathrm{C} 136-\mathrm{H} 13 \mathrm{~L}$ & 0.9700 \\
\hline $\mathrm{C} 24-\mathrm{C} 25$ & $1.382(5)$ & $\mathrm{O} 1 \mathrm{~S}-\mathrm{C} 2 \mathrm{~S}$ & $1.174(8)$ \\
\hline $\mathrm{C} 24-\mathrm{C} 26^{\mathrm{ii}}$ & $1.376(5)$ & $\mathrm{N} 3 \mathrm{~S}-\mathrm{C} 2 \mathrm{~S}$ & $1.208(9)$ \\
\hline $\mathrm{C} 25-\mathrm{H} 25$ & 0.9300 & $\mathrm{~N} 3 \mathrm{~S}-\mathrm{C} 4 \mathrm{~S}$ & $1.393(8)$ \\
\hline $\mathrm{C} 25-\mathrm{C} 26$ & $1.363(5)$ & $\mathrm{N} 3 \mathrm{~S}-\mathrm{C} 5 \mathrm{~S}$ & $1.432(10)$ \\
\hline $\mathrm{C} 26-\mathrm{C} 24^{\mathrm{ii}}$ & $1.376(5)$ & $\mathrm{C} 2 \mathrm{~S}-\mathrm{H} 2 \mathrm{~S}$ & 0.9300 \\
\hline $\mathrm{C} 26-\mathrm{H} 26$ & 0.9300 & $\mathrm{C} 4 \mathrm{~S}-\mathrm{H} 4 \mathrm{SA}$ & 0.9600 \\
\hline $\mathrm{C} 33-\mathrm{C} 34$ & $1.508(5)$ & $\mathrm{C} 4 \mathrm{~S}-\mathrm{H} 4 \mathrm{SB}$ & 0.9600 \\
\hline $\mathrm{C} 34-\mathrm{C} 35$ & $1.383(5)$ & $\mathrm{C} 4 \mathrm{~S}-\mathrm{H} 4 \mathrm{SC}$ & 0.9600 \\
\hline $\mathrm{C} 34-\mathrm{C} 36^{\mathrm{iii}}$ & $1.374(5)$ & $\mathrm{C} 5 \mathrm{~S}-\mathrm{H} 5 \mathrm{SA}$ & 0.9600 \\
\hline $\mathrm{C} 35-\mathrm{H} 35$ & 0.9300 & C5S-H5SB & 0.9600 \\
\hline $\mathrm{C} 35-\mathrm{C} 36$ & $1.379(5)$ & $\mathrm{C} 5 \mathrm{~S}-\mathrm{H} 5 \mathrm{SC}$ & 0.9600 \\
\hline
\end{tabular}




\begin{tabular}{|c|c|c|c|}
\hline $\mathrm{C} 36-\mathrm{C} 34^{\mathrm{iii}}$ & $1.374(5)$ & & \\
\hline $\mathrm{O} 21-\mathrm{Zn} 1-\mathrm{O} 31$ & $105.00(12)$ & $\mathrm{N} 124-\mathrm{C} 125-\mathrm{N} 121$ & $110.0(4)$ \\
\hline $\mathrm{O} 21-\mathrm{Zn} 1-\mathrm{N} 111$ & $124.42(12)$ & $\mathrm{N} 124-\mathrm{C} 125-\mathrm{H} 125$ & 125.0 \\
\hline $\mathrm{O} 21-\mathrm{Zn} 1-\mathrm{N} 121^{\mathrm{i}}$ & $104.47(12)$ & $\mathrm{N} 114-\mathrm{C} 131-\mathrm{H} 13 \mathrm{~A}$ & 108.7 \\
\hline $\mathrm{O} 31-\mathrm{Zn} 1-\mathrm{N} 111$ & $118.44(13)$ & $\mathrm{N} 114-\mathrm{C} 131-\mathrm{H} 13 \mathrm{~B}$ & 108.7 \\
\hline $\mathrm{O} 31-\mathrm{Zn} 1-\mathrm{N} 121^{\mathrm{i}}$ & $98.53(12)$ & $\mathrm{N} 114-\mathrm{C} 131-\mathrm{C} 132$ & $114.2(4)$ \\
\hline $\mathrm{N} 111-\mathrm{Zn} 1-\mathrm{N} 121^{\mathrm{i}}$ & $101.66(12)$ & $\mathrm{H} 13 \mathrm{~A}-\mathrm{C} 131-\mathrm{H} 13 \mathrm{~B}$ & 107.6 \\
\hline $\mathrm{C} 23-\mathrm{O} 21-\mathrm{Zn} 1$ & $110.9(2)$ & $\mathrm{C} 132-\mathrm{C} 131-\mathrm{H} 13 \mathrm{~A}$ & 108.7 \\
\hline $\mathrm{C} 33-\mathrm{O} 31-\mathrm{Zn} 1$ & $110.0(3)$ & $\mathrm{C} 132-\mathrm{C} 131-\mathrm{H} 13 \mathrm{~B}$ & 108.7 \\
\hline $\mathrm{C} 112-\mathrm{N} 111-\mathrm{Zn} 1$ & $131.6(3)$ & $\mathrm{C} 131-\mathrm{C} 132-\mathrm{H} 13 \mathrm{C}$ & 109.6 \\
\hline $\mathrm{C} 115-\mathrm{N} 111-\mathrm{Zn} 1$ & $126.1(3)$ & $\mathrm{C} 131-\mathrm{C} 132-\mathrm{H} 13 \mathrm{D}$ & 109.6 \\
\hline $\mathrm{C} 115-\mathrm{N} 111-\mathrm{C} 112$ & $102.1(3)$ & $\mathrm{C} 131-\mathrm{C} 132-\mathrm{C} 133$ & $110.3(4)$ \\
\hline $\mathrm{C} 112-\mathrm{N} 113-\mathrm{N} 114$ & $102.6(3)$ & $\mathrm{H} 13 \mathrm{C}-\mathrm{C} 132-\mathrm{H} 13 \mathrm{D}$ & 108.1 \\
\hline N113-N114-C131 & $119.8(3)$ & $\mathrm{C} 133-\mathrm{C} 132-\mathrm{H} 13 \mathrm{C}$ & 109.6 \\
\hline C115-N114-N113 & $109.4(3)$ & $\mathrm{C} 133-\mathrm{C} 132-\mathrm{H} 13 \mathrm{D}$ & 109.6 \\
\hline $\mathrm{C} 115-\mathrm{N} 114-\mathrm{C} 131$ & $130.7(4)$ & $\mathrm{C} 132-\mathrm{C} 133-\mathrm{H} 13 \mathrm{E}$ & 108.5 \\
\hline $\mathrm{C} 122-\mathrm{N} 121-\mathrm{Zn} 1^{\mathrm{i}}$ & $128.6(3)$ & $\mathrm{C} 132-\mathrm{C} 133-\mathrm{H} 13 \mathrm{~F}$ & 108.5 \\
\hline $\mathrm{C} 125-\mathrm{N} 121-\mathrm{Zn} 1^{\mathrm{i}}$ & $128.2(3)$ & $\mathrm{C} 132-\mathrm{C} 133-\mathrm{C} 134$ & $115.3(4)$ \\
\hline $\mathrm{C} 125-\mathrm{N} 121-\mathrm{C} 122$ & $102.5(3)$ & $\mathrm{H} 13 \mathrm{E}-\mathrm{C} 133-\mathrm{H} 13 \mathrm{~F}$ & 107.5 \\
\hline $\mathrm{C} 122-\mathrm{N} 123-\mathrm{N} 124$ & $102.6(3)$ & $\mathrm{C} 134-\mathrm{C} 133-\mathrm{H} 13 \mathrm{E}$ & 108.5 \\
\hline $\mathrm{N} 123-\mathrm{N} 124-\mathrm{C} 136$ & $121.4(3)$ & $\mathrm{C} 134-\mathrm{C} 133-\mathrm{H} 13 \mathrm{~F}$ & 108.5 \\
\hline $\mathrm{C} 125-\mathrm{N} 124-\mathrm{N} 123$ & $110.2(3)$ & $\mathrm{C} 133-\mathrm{C} 134-\mathrm{H} 13 \mathrm{G}$ & 109.4 \\
\hline $\mathrm{C} 125-\mathrm{N} 124-\mathrm{C} 136$ & $128.4(4)$ & $\mathrm{C} 133-\mathrm{C} 134-\mathrm{H} 13 \mathrm{H}$ & 109.4 \\
\hline $\mathrm{O} 21-\mathrm{C} 23-\mathrm{C} 24$ & $116.7(3)$ & $\mathrm{H} 13 \mathrm{G}-\mathrm{C} 134-\mathrm{H} 13 \mathrm{H}$ & 108.0 \\
\hline $\mathrm{O} 22-\mathrm{C} 23-\mathrm{O} 21$ & $123.9(4)$ & $\mathrm{C} 135-\mathrm{C} 134-\mathrm{C} 133$ & $111.3(4)$ \\
\hline $\mathrm{O} 22-\mathrm{C} 23-\mathrm{C} 24$ & $119.4(3)$ & $\mathrm{C} 135-\mathrm{C} 134-\mathrm{H} 13 \mathrm{G}$ & 109.4 \\
\hline $\mathrm{C} 25-\mathrm{C} 24-\mathrm{C} 23$ & $121.5(4)$ & $\mathrm{C} 135-\mathrm{C} 134-\mathrm{H} 13 \mathrm{H}$ & 109.4 \\
\hline $\mathrm{C} 26^{\mathrm{ii}}-\mathrm{C} 24-\mathrm{C} 23$ & $121.3(3)$ & $\mathrm{C} 134-\mathrm{C} 135-\mathrm{H} 13 \mathrm{I}$ & 108.1 \\
\hline $\mathrm{C} 26^{\mathrm{ii}}-\mathrm{C} 24-\mathrm{C} 25$ & $117.1(3)$ & $\mathrm{C} 134-\mathrm{C} 135-\mathrm{H} 13 \mathrm{~J}$ & 108.1 \\
\hline $\mathrm{C} 24-\mathrm{C} 25-\mathrm{H} 25$ & 119.2 & $\mathrm{H} 13 \mathrm{I}-\mathrm{C} 135-\mathrm{H} 13 \mathrm{~J}$ & 107.3 \\
\hline $\mathrm{C} 26-\mathrm{C} 25-\mathrm{C} 24$ & $121.7(4)$ & $\mathrm{C} 136-\mathrm{C} 135-\mathrm{C} 134$ & $116.8(4)$ \\
\hline $\mathrm{C} 26-\mathrm{C} 25-\mathrm{H} 25$ & 119.2 & $\mathrm{C} 136-\mathrm{C} 135-\mathrm{H} 13 \mathrm{I}$ & 108.1 \\
\hline $\mathrm{C} 24^{\mathrm{ii}}-\mathrm{C} 26-\mathrm{H} 26$ & 119.4 & $\mathrm{C} 136-\mathrm{C} 135-\mathrm{H} 13 \mathrm{~J}$ & 108.1 \\
\hline $\mathrm{C} 25-\mathrm{C} 26-\mathrm{C} 24^{\mathrm{ii}}$ & $121.2(4)$ & $\mathrm{N} 124-\mathrm{C} 136-\mathrm{C} 135$ & $113.0(3)$ \\
\hline $\mathrm{C} 25-\mathrm{C} 26-\mathrm{H} 26$ & 119.4 & $\mathrm{~N} 124-\mathrm{C} 136-\mathrm{H} 13 \mathrm{~K}$ & 109.0 \\
\hline $\mathrm{O} 31-\mathrm{C} 33-\mathrm{C} 34$ & $117.3(4)$ & N124-C136-H13L & 109.0 \\
\hline $\mathrm{O} 32-\mathrm{C} 33-\mathrm{O} 31$ & $123.2(4)$ & $\mathrm{C} 135-\mathrm{C} 136-\mathrm{H} 13 \mathrm{~K}$ & 109.0 \\
\hline $\mathrm{O} 32-\mathrm{C} 33-\mathrm{C} 34$ & $119.4(4)$ & $\mathrm{C} 135-\mathrm{C} 136-\mathrm{H} 13 \mathrm{~L}$ & 109.0 \\
\hline $\mathrm{C} 35-\mathrm{C} 34-\mathrm{C} 33$ & $120.5(3)$ & $\mathrm{H} 13 \mathrm{~K}-\mathrm{C} 136-\mathrm{H} 13 \mathrm{~L}$ & 107.8 \\
\hline 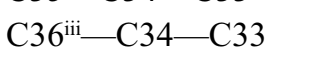 & $120.8(3)$ & $\mathrm{C} 2 \mathrm{~S}-\mathrm{N} 3 \mathrm{~S}-\mathrm{C} 4 \mathrm{~S}$ & $130.5(8)$ \\
\hline $\mathrm{C} 36^{\mathrm{iii}-\mathrm{C} 34-\mathrm{C} 35}$ & $118.7(3)$ & $\mathrm{C} 2 \mathrm{~S}-\mathrm{N} 3 \mathrm{~S}-\mathrm{C} 5 \mathrm{~S}$ & $116.7(7)$ \\
\hline C34-C35-H35 & 119.8 & $\mathrm{C} 4 \mathrm{~S}-\mathrm{N} 3 \mathrm{~S}-\mathrm{C} 5 \mathrm{~S}$ & $112.0(6)$ \\
\hline $\mathrm{C} 36-\mathrm{C} 35-\mathrm{C} 34$ & $120.4(3)$ & $\mathrm{O} 1 \mathrm{~S}-\mathrm{C} 2 \mathrm{~S}-\mathrm{N} 3 \mathrm{~S}$ & $134.7(10)$ \\
\hline $\mathrm{C} 36-\mathrm{C} 35-\mathrm{H} 35$ & 119.8 & $\mathrm{O} 1 \mathrm{~S}-\mathrm{C} 2 \mathrm{~S}-\mathrm{H} 2 \mathrm{~S}$ & 112.7 \\
\hline $\mathrm{C} 34^{\mathrm{iii}-\mathrm{C} 36-\mathrm{C} 35}$ & $120.8(3)$ & $\mathrm{N} 3 \mathrm{~S}-\mathrm{C} 2 \mathrm{~S}-\mathrm{H} 2 \mathrm{~S}$ & 112.7 \\
\hline $\mathrm{C} 34^{\mathrm{iii}-\mathrm{C} 36-\mathrm{H} 36}$ & 119.6 & $\mathrm{~N} 3 \mathrm{~S}-\mathrm{C} 4 \mathrm{~S}-\mathrm{H} 4 \mathrm{SA}$ & 109.5 \\
\hline $\mathrm{C} 35-\mathrm{C} 36-\mathrm{H} 36$ & 119.6 & $\mathrm{~N} 3 \mathrm{~S}-\mathrm{C} 4 \mathrm{~S}-\mathrm{H} 4 \mathrm{SB}$ & 109.5 \\
\hline
\end{tabular}




\begin{tabular}{|c|c|}
\hline $\mathrm{N} 111-\mathrm{C} 112-\mathrm{H} 112$ & 122.6 \\
\hline $\mathrm{N} 113-\mathrm{C} 112-\mathrm{N} 111$ & $114.8(4)$ \\
\hline $\mathrm{N} 113-\mathrm{C} 112-\mathrm{H} 112$ & 122.6 \\
\hline $\mathrm{N} 111-\mathrm{C} 115-\mathrm{H} 115$ & 124.4 \\
\hline $\mathrm{N} 114-\mathrm{C} 115-\mathrm{N} 111$ & $111.1(4)$ \\
\hline N114-C115-H115 & 124.4 \\
\hline $\mathrm{N} 121-\mathrm{C} 122-\mathrm{H} 122$ & 122.6 \\
\hline $\mathrm{N} 123-\mathrm{C} 122-\mathrm{N} 121$ & $114.8(4)$ \\
\hline $\mathrm{N} 123-\mathrm{C} 122-\mathrm{H} 122$ & 122.6 \\
\hline $\mathrm{N} 121-\mathrm{C} 125-\mathrm{H} 125$ & 125.0 \\
\hline $\mathrm{Zn} 1-\mathrm{O} 21-\mathrm{C} 23-\mathrm{O} 22$ & $8.3(5)$ \\
\hline $\mathrm{Zn} 1-\mathrm{O} 21-\mathrm{C} 23-\mathrm{C} 24$ & $-170.1(2)$ \\
\hline $\mathrm{Zn} 1-\mathrm{O} 31-\mathrm{C} 33-\mathrm{O} 32$ & $-0.1(5)$ \\
\hline $\mathrm{Zn} 1-\mathrm{O} 31-\mathrm{C} 33-\mathrm{C} 34$ & $-176.8(3)$ \\
\hline $\mathrm{Zn} 1-\mathrm{N} 111-\mathrm{C} 112-\mathrm{N} 113$ & $-176.0(3)$ \\
\hline Zn1-N111-C115-N114 & $176.7(3)$ \\
\hline $\mathrm{Zn} 1 \mathrm{i}-\mathrm{N} 121-\mathrm{C} 122-\mathrm{N} 123$ & $170.8(3)$ \\
\hline $\mathrm{Zn} 1{ }^{\mathrm{i}}-\mathrm{N} 121-\mathrm{C} 125-\mathrm{N} 124$ & $-171.3(2)$ \\
\hline $\mathrm{O} 21-\mathrm{C} 23-\mathrm{C} 24-\mathrm{C} 25$ & $-175.2(3)$ \\
\hline $\mathrm{O} 21-\mathrm{C} 23-\mathrm{C} 24-\mathrm{C} 26^{\mathrm{ii}}$ & $5.7(5)$ \\
\hline $\mathrm{O} 22-\mathrm{C} 23-\mathrm{C} 24-\mathrm{C} 25$ & $6.4(5)$ \\
\hline $\mathrm{O} 22-\mathrm{C} 23-\mathrm{C} 24-\mathrm{C} 26^{\mathrm{ii}}$ & $-172.7(4)$ \\
\hline $\mathrm{O} 31-\mathrm{C} 33-\mathrm{C} 34-\mathrm{C} 35$ & $175.8(4)$ \\
\hline $\mathrm{O} 31-\mathrm{C} 33-\mathrm{C} 34-\mathrm{C} 36^{\mathrm{iii}}$ & $-3.0(6)$ \\
\hline $\mathrm{O} 32-\mathrm{C} 33-\mathrm{C} 34-\mathrm{C} 35$ & $-1.0(6)$ \\
\hline $\mathrm{O} 32-\mathrm{C} 33-\mathrm{C} 34-\mathrm{C} 36^{\mathrm{iii}}$ & $-179.8(4)$ \\
\hline N113-N114-C115-N111 & $-0.6(5)$ \\
\hline N113-N114-C131-C132 & $149.1(4)$ \\
\hline N114-N113-C112-N111 & $-0.4(5)$ \\
\hline $\mathrm{N} 114-\mathrm{C} 131-\mathrm{C} 132-\mathrm{C} 133$ & $174.5(4)$ \\
\hline $\mathrm{N} 123-\mathrm{N} 124-\mathrm{C} 125-\mathrm{N} 121$ & $0.4(4)$ \\
\hline $\mathrm{N} 123-\mathrm{N} 124-\mathrm{C} 136-\mathrm{C} 135$ & $-99.8(5)$ \\
\hline $\mathrm{N} 124-\mathrm{N} 123-\mathrm{C} 122-\mathrm{N} 121$ & $0.7(5)$ \\
\hline $\mathrm{C} 23-\mathrm{C} 24-\mathrm{C} 25-\mathrm{C} 26$ & $-179.3(4)$ \\
\hline
\end{tabular}

$\begin{array}{ll}\text { N3S-C4S-H4SC } & 109.5 \\ \text { H4SA-C4S-H4SB } & 109.5 \\ \text { H4SA-C4S-H4SC } & 109.5 \\ \text { H4SB-C4S-H4SC } & 109.5 \\ \text { N3S-C5S-H5SA } & 109.5 \\ \text { N3S-C5S-H5SB } & 109.5 \\ \text { N3S-C5S-H5SC } & 109.5 \\ \text { H5SA-C5S-H5SB } & 109.5 \\ \text { H5SA-C5S-H5SC } & 109.5 \\ \text { H5SB-C5S-H5SC } & 109.5\end{array}$

$\mathrm{C} 24-\mathrm{C} 25-\mathrm{C} 26-\mathrm{C} 24^{\mathrm{ii}} \quad 0.1(7)$

$\mathrm{C} 26^{\mathrm{ii}}-\mathrm{C} 24-\mathrm{C} 25-\mathrm{C} 26 \quad-0.1(7)$

$\mathrm{C} 33-\mathrm{C} 34-\mathrm{C} 35-\mathrm{C} 36 \quad-178.3$ (4)

$\mathrm{C} 34-\mathrm{C} 35-\mathrm{C} 36-\mathrm{C} 34^{\mathrm{iii}} \quad-0.5(7)$

$\mathrm{C} 36{ }^{\mathrm{iii}}-\mathrm{C} 34-\mathrm{C} 35-\mathrm{C} 36 \quad 0.5(7)$

C112-N111-C115-N114 0.3 (5)

$\mathrm{C} 112-\mathrm{N} 113-\mathrm{N} 114-\mathrm{C} 115 \quad 0.6(5)$

C112-N113-N114-C131 176.1 (4)

C115-N111-C112-N113 $0.0(5)$

$\mathrm{C} 115-\mathrm{N} 114-\mathrm{C} 131-\mathrm{C} 132 \quad-36.4$ (7)

$\mathrm{C} 122-\mathrm{N} 121-\mathrm{C} 125-\mathrm{N} 124 \quad 0.1(4)$

$\mathrm{C} 122-\mathrm{N} 123-\mathrm{N} 124-\mathrm{C} 125 \quad-0.6(4)$

$\mathrm{C} 122-\mathrm{N} 123-\mathrm{N} 124-\mathrm{C} 136 \quad-178.1$ (3)

$\mathrm{C} 125-\mathrm{N} 121-\mathrm{C} 122-\mathrm{N} 123-0.5(5)$

$\mathrm{C} 125-\mathrm{N} 124-\mathrm{C} 136-\mathrm{C} 135 \quad 83.2(5)$

C131-N114-C115-N111 - 175.5 (4)

$\mathrm{C} 131-\mathrm{C} 132-\mathrm{C} 133-\mathrm{C} 134-175.0(4)$

$\mathrm{C} 132-\mathrm{C} 133-\mathrm{C} 134-\mathrm{C} 135 \quad 177.6(3)$

$\mathrm{C} 133-\mathrm{C} 134-\mathrm{C} 135-\mathrm{C} 136 \quad-177.4$ (3)

$\mathrm{C} 134-\mathrm{C} 135-\mathrm{C} 136-\mathrm{N} 124 \quad 66.5$ (5)

$\mathrm{C} 136-\mathrm{N} 124-\mathrm{C} 125-\mathrm{N} 121 \quad 177.7$ (3)

$\mathrm{C} 4 \mathrm{~S}-\mathrm{N} 3 \mathrm{~S}-\mathrm{C} 2 \mathrm{~S}-\mathrm{O} 1 \mathrm{~S} \quad-177.8(11)$

$\mathrm{C} 5 \mathrm{~S}-\mathrm{N} 3 \mathrm{~S}-\mathrm{C} 2 \mathrm{~S}-\mathrm{O} 1 \mathrm{~S} \quad-9.0(19)$

Symmetry codes: (i) $-x+2,-y+2,-z+1$; (ii) $-x+2,-y+1,-z$; (iii) $-x,-y,-z$.

Hydrogen-bond geometry $\left(\AA,{ }^{\circ}\right)$

$\mathrm{Cg}$ is the centroid of the $\mathrm{C} 24-\mathrm{C} 26 / \mathrm{C} 24^{\mathrm{i}}-\mathrm{C} 26^{\mathrm{i}}$ ring.

\begin{tabular}{lllll}
\hline$D-\mathrm{H} \cdots A$ & $D-\mathrm{H}$ & $\mathrm{H} \cdots A$ & $D \cdots A$ & $D-\mathrm{H} \cdots A$ \\
\hline $\mathrm{C} 36^{\text {iv }}-\mathrm{H} 36^{\text {iv } \cdots} C g$ & 0.93 & 3.07 & 3.95 & 149 \\
\hline
\end{tabular}

Symmetry code: (iv) $x+1, y+1, z$. 